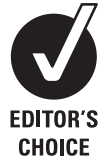

Nuclear Cardiology, Ramistrasse 100, University Hospital Zurich, 8091 Zurich, Switzerland; ${ }^{2}$ Zurich Center for Integrative Human Physiology (ZIHP), University of Zurich, Switzerland

Correspondence to: Professor Philipp A Kaufmann, Nuclear Cardiology, University Hospital Zurich, Ramistrasse 100, CH-8091 Zurich.

Switzerland; pak@usz.ch

BAH and CAW contributed equally to this work.

Accepted 15 June 2009 Published Online First 5 July 2009

\title{
First head-to-head comparison of effective radiation dose from low-dose 64-slice CT with prospective ECG-triggering versus invasive coronary angiography
}

\author{
B A Herzog, ${ }^{1}$ C A Wyss, ${ }^{1}$ L Husmann, ${ }^{1} 0$ Gaemperli, ${ }^{1}$ I Valenta, ${ }^{1}$ V Treyer, \\ U Landmesser, ${ }^{1}$ P A Kaufmann ${ }^{1,2}$
}

\begin{abstract}
Background: Reduction of radiation burden of multidetector computed tomography coronary angiography (CTCA) has remained an important task.

Objective: To compare effective radiation dose of lowdose 64-slice CTCA using prospective ECG-triggering versus diagnostic invasive coronary angiography (CA). Methods: 42 patients referred for elective invasive CA owing to suspected coronary artery disease (CAD) were prospectively enrolled to undergo a low-dose CTCA without calcium scoring within the same day before CA. Dose-area product of diagnostic invasive CA and doselength product of CTCA were measured, converted into effective radiation dose and compared using MannWhitney $U$ tests. In addition, accuracy of CTCA to detect CAD (coronary artery narrowing $\geqslant 50 \%$ ) was assessed using invasive CA as standard of reference. On an intention-to-diagnose basis all non-evaluative vessels were included in the analysis and censored as positive. Results: The estimated mean effective radiation dose was 8.5 (4.4) mSv (range 1.4-20.5 mSv) for diagnostic invasive CA, and 2.1 (0.7) $\mathrm{mSv}$ (range 1.0-3.3 mSv) for CTCA ( $p<0.001) .19$ patients $(42.9 \%)$ had no CAD by invasive CA. 40 (95.2\%) patients have been correctly classified as having CAD (23/23) or no CAD (17/19). Over $97 \%(551 / 567)$ of segments were evaluable. Vesselbased analysis revealed sensitivity, specificity, positive and negative predictive value of $94.2 \%(\mathrm{Cl} 0.8 \%$ to $1.0 \%)$, $94.8 \%$ (Cl $09 \%$ to $1.0 \%$ ), $89.0 \%$ (Cl $0.8 \%$ to $1.0 \%$ ), $97.4 \%$ (Cl $09 \%$ to $1.0 \%$ ) and an accuracy of $94.6 \%$.

Conclusions: Low dose CTCA allows evaluation of CAD with high accuracy, but delivers a significantly less effective radiation dose to patients compared to diagnostic invasive CA.
\end{abstract}

Since the implementation of 64-slice computed tomography (CT) scanners, CT coronary angiography (CTCA) has developed as a non-invasive tool with high accuracy for the evaluation of coronary artery disease (CAD). ${ }^{1}$ However, effective radiation dose $(E)$ to patients and its risk of cancer induction have remained issues of concern, although only few quantitative data on the doserisk relation are available. ${ }^{2}$ The latter is mainly based on epidemiological studies from atomic bomb survivors from the Hiroshima and Nagasaki tumour registries, studies of people exposed for medical reasons and studies of nuclear workers. ${ }^{3}$ The risk of cancer from exposure to low levels of ionising radiation, defined as effective dose below $1 \mathrm{mSv}$ and up to $100 \mathrm{mSv}$, may proceed in a linear fashion to increasing radiation dose without a lower threshold. Thus, small radiation doses to patients may cause a small increase in risk. ${ }^{3}$ The American Heart Association states that a CT with an effective radiation dose of about $10 \mathrm{mSv}$ may be associated with an increase in the possibility of fatal cancer. ${ }^{4}$ Brenner et al estimated a lifetime cancer mortality risk of $0.08 \%$ attributable to a full-body CT scan with an effective radiation dose of $12 \mathrm{mSv}$ in a 45-year-old male adult. ${ }^{5}$ Although this may appear justifiable under certain clinical circumstances the risk may be higher in younger patients and female patients. Therefore, any successful reduction of radiation dose is important. By using ECG-modulation the radiation burden of CTCA could be reduced from initially over $20 \mathrm{mSv}^{6}$ to $10-15 \mathrm{mSv}{ }^{7}$ and with optimised protocols below $8 \mathrm{mSv}^{8}$ A milestone in the reduction of $E$ could be achieved by a new scanning protocol using prospective ECG-triggering where radiation is administered at only one predefined end-diastolic time point instead of during a whole phase of the cardiac cycle. ${ }^{9}$ First reports demonstrated the feasibility ${ }^{1011}$ and latest results confirm maintained high diagnostic accuracy ${ }^{12}$ of this new technique.

Although the United Nations Scientific Committee on the Effects of Atomic Radiation cites a typical value of about $7 \mathrm{mSv}$ for $E$ from invasive $\mathrm{CA},{ }^{14}$ no data on direct head-to-head comparison in the same patient between $E$ from CTCA and CA are available.

The aim of the present study was to assess $E$ of diagnostic invasive CA and low dose CTCA using prospective ECG-triggering in the same patient.

\section{PATIENTS AND METHODS \\ Study population}

Among 74 consecutively screened patients with no known $\mathrm{CAD}$, nine refused to give consent and could not be included. Of the 65 enrolled patients 14 were deemed ineligible due to renal insufficiency (creatinine levels $>150 \mu \mathrm{mol} / \mathrm{l}$, or $>1.7 \mathrm{mg} / \mathrm{dl}$ ) $(\mathrm{n}=8)$; allergy to iodinated contrast agent $(\mathrm{n}=3)$, non-sinus rhythm or known premature ventricular or supraventricular beats $(n=12)$. The final study population consisted of 42 prospectively enrolled patients, different from patients included in previously reported studies on the feasibility ${ }^{11}$ and accuracy ${ }^{12}$ of low dose CTCA with prospective ECG-triggering. The pre-test probability for CAD was estimated using the Duke clinical score, which includes the type of chest discomfort, age, gender and traditional risk factors. ${ }^{15}$ All patients were referred for elective invasive CA 


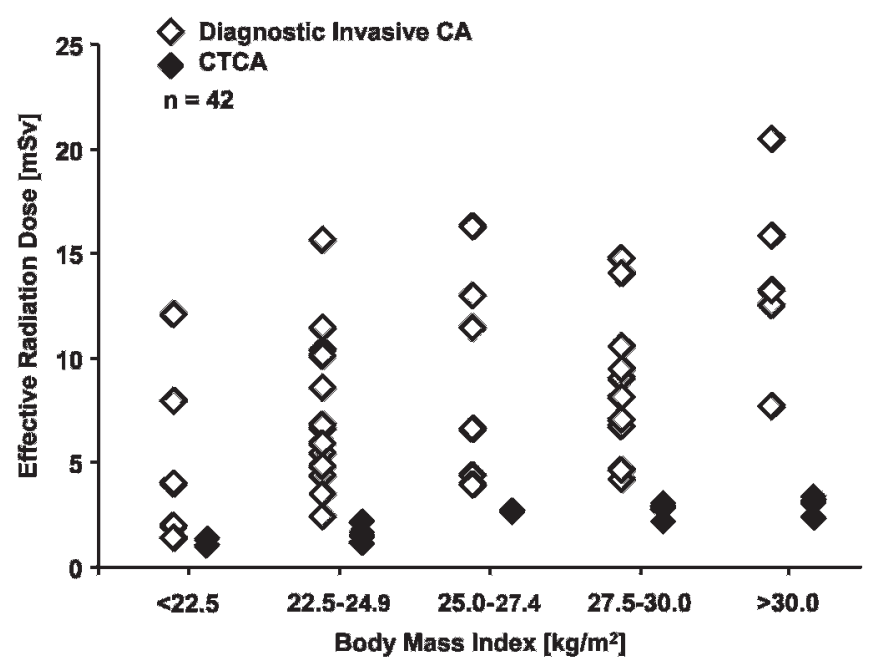

Figure 1 Effective radiation doses for each patient from the two different methods - that is, diagnostic invasive coronary angiography (CA) and CT coronary angiography (CTCA).

because of suspected CAD with the following symptoms: dyspnoea $(n=9)$, typical angina pectoris $(n=7)$, atypical chest pain $(n=19)$ and pathological exercise test or ECG $(n=14)$. CTCA and invasive CA were successfully performed in all 42 patients (29 male, 13 female; age 62 (8.4) years, range 42-82 years) on the same day. Thirteen patients were on $\beta$-blocker therapy as part of their baseline medication. Additional intravenous metoprolol (2-20 mg) (Beloc, AstraZeneca, London, UK) was administered for heart rate control before CTCA in 22 patients (mean $5.7(6.6) \mathrm{mg}$ ) yielding a mean heart rate of 55.4 (6.2) bpm (range 36-70 bpm) and a mean heart rate variability of $1.6(1.7) \mathrm{bpm}$ (range $0.3-9.7 \mathrm{bpm}$ ). The mean body mass index (BMI) was $26.9(4.4) \mathrm{kg} / \mathrm{m}^{2}$ (range 18.6-44.8 kg/m²). The study protocol was approved by the local ethics committee and written informed consent was obtained.

\section{Data acquisition and post-processing}

All patients received a single dose of $2.5 \mathrm{mg}$ isosorbide dinitrate sublingually (Isoket, Schwarz Pharma, Monheim, Germany) 2 minutes before the scan. In addition, intravenous metoprolol was administered, if necessary to achieve a target heart below $65 \mathrm{bpm}$ before the start of the scan. Heart rate was monitored and heart rate variability was assessed as the standard deviation of the heart rate throughout the scan as previously reported. ${ }^{16}$ For CTCA, $80 \mathrm{ml}$ of iodixanol (Visipaque 320, $320 \mathrm{mg} / \mathrm{ml}$, GE Heathcare, Bucks, UK) at a flow rate of $5 \mathrm{ml} / \mathrm{s}$ followed by $50 \mathrm{ml}$ saline solution was injected into an antecubital vein via an 18-gauge catheter. Bolus tracking was performed with a region of interest placed into the ascending aorta, and image acquisition was started 4 seconds after the signal density reached a threshold of approximately 120 Hounsfield units. All CTCA examinations were performed with a LightSpeed VCT XT scanner (GE Healthcare) and prospective ECGtriggering 9 using a commercially available protocol (SnapShot Pulse, GE Healthcare) and the following scanning parameters as previously reported ${ }^{11}$ : slice acquisition acquisition $64 \mathrm{~mm} \times$ $0.625 \mathrm{~mm}$, smallest $\mathrm{x}$-ray window (only $75 \%$ of the RR-cycle, setting the padding to $0 \mathrm{~ms}$ ), z-coverage $40 \mathrm{~mm}$ with an increment of $35 \mathrm{~mm}$, gantry rotation time $350 \mathrm{~ms}$, BMI adapted tube voltage (100 kV: BMI $<25 \mathrm{~kg} / \mathrm{m}^{2}, 120 \mathrm{kV}$ : BMI $\geqslant 25 \mathrm{~kg} / \mathrm{m}^{2}$ ) and effective tube-current (450 mA: BMI $<22.5 \mathrm{~kg} / \mathrm{m}^{2}, 500 \mathrm{~mA}$ :
BMI 22.5-24.9 kg/m², $550 \mathrm{~mA}$ : BMI $25.0-27.4 \mathrm{~kg} / \mathrm{m}^{2}, 600 \mathrm{~mA}$ : BMI $27.5-30 \mathrm{~kg} / \mathrm{m}^{2}, 650 \mathrm{~mA}$ : BMI $\left.>30 \mathrm{~kg} / \mathrm{m}^{2}\right)$. The CT scan was performed from below the tracheal bifurcation to the diaphragm, choosing three to four scan blocks (field of view 11$14.5 \mathrm{~cm}$ ). By restricting the scan to the smallest possible window at only one distinct end-diastolic phase of the RRcycle (that is, $75 \%$ ) we ascertained the lowest achievable effective dose delivery. CTCA images were reconstructed with a slice thickness of $0.6 \mathrm{~mm}$, using a medium-soft tissue convolution kernel (standard). In case of vessel wall calcifications, additional images were reconstructed using a sharp-tissue convolution kernel (detail) and preferably analysed using a bone window setting (window width: $1500 \mathrm{HU}$; window level: $500 \mathrm{HU}$ ) to compensate for blooming artefacts. All images were transferred to an external workstation (AW 4.4, GE Healthcare).

\section{CT image analysis}

Coronary arteries were segmented as suggested by the American Heart Association. ${ }^{17}$ The right coronary artery (RCA) was defined to include segments $1-4$, the left main artery (LMA) to include segment 5 , the left anterior descending artery (LAD) to include segments $6-10$, and the left circumflex artery (CX) to include segments 11-16; the intermediate artery was designated as segment 16, if present. All segments with a diameter of at least $1.5 \mathrm{~mm}$ at their origin were included. Step artefacts at junctions of different image blocks may not necessarily lead to misinterpretations. However, as a hidden lesion within the artefact cannot be excluded with absolute certainty we have categorised any step artefact as non-evaluative. On an intention-to-diagnose basis all non-evaluative segments classified the whole vessel as not evaluative which was censored as positive and included in the final analysis. Two readers experienced in cardiac radiology assessed all coronary vessels for the presence of haemodynamically significant stenoses, defined as narrowing of

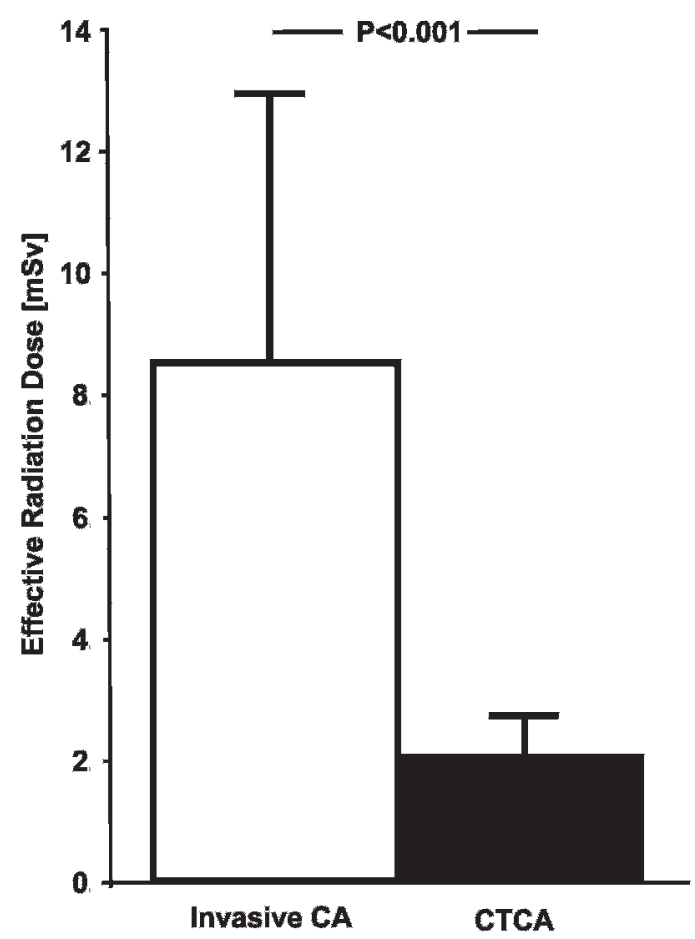

Figure 2 Comparison of mean effective radiation dose (SD) of diagnostic invasive coronary angiography (CA) and CT coronary angiography (CTCA). 
Table 1 Effective radiation dose: CTCA versus invasive CA

\begin{tabular}{|c|c|c|c|c|c|c|c|c|}
\hline \multirow[b]{2}{*}{ BMI } & \multicolumn{5}{|c|}{ CTCA } & \multicolumn{2}{|l|}{ CA } & \multirow[b]{2}{*}{ p Value } \\
\hline & No & Current $(\mathrm{mA})$ & Voltage (kV) & $\operatorname{DLP}(\mathbf{m G y} \times \mathbf{c m})$ & $\begin{array}{l}\text { Radiation dose } \\
\text { (mSv) }\end{array}$ & DAP $\left(\mathbf{m G y} \times \mathbf{c m}^{2}\right)$ & Radiation dose (mSv) & \\
\hline All patients & 42 & & & $122.4(42.1)$ & $2.1(0.7)$ & $38.7(20.1)$ & $8.5(4.4)$ & $<0.001^{*}$ \\
\hline $22.5-24.9 \mathrm{~kg} / \mathrm{m}^{2}$ & 15 & 500 & 100 & $86.5(13.3)$ & $1.5(0.2)$ & $33.0(15.6)$ & $7.3(3.4)$ & $<0.001^{*}$ \\
\hline $25-27.4 \mathrm{~kg} / \mathrm{m}^{2}$ & 6 & 550 & 120 & $152.6(0.3)$ & $2.6(0.0)$ & $42.2(22.9)$ & $9.3(5.0)$ & $0.002^{*}$ \\
\hline $27.5-30 \mathrm{~kg} / \mathrm{m}^{2}$ & 11 & 600 & 120 & $155.4(19.7)$ & $2.6(0.3)$ & $39.5(15.5)$ & $8.7(3.4)$ & $<0.001^{*}$ \\
\hline
\end{tabular}

*Bonferroni-adjusted level of significance was $p<0.0083$.

$\mathrm{BMI}$, body mass index; CTCA, computed tomography coronary angiography; DAP, dose-area product; DLP, dose-length product; ICA, invasive coronary angiography.

the coronary luminal diameter $\geqslant 50 \%$. In case of multiple lesions in one segment, the segment was classified by the worst lesion. For any disagreement in data analysis between the two observers, consensus agreement was achieved.

\section{Invasive coronary angiography}

Invasive CA through the femoral artery was performed on an Allura 9 and an Allura XPER FD10/10 (Philips Medical Systems) catheterisation equipment in an experienced catheterisation laboratory of a teaching hospital (University Hospital Zurich) following a protocol, which consists of a biplane angiography of the left coronary artery with two radiation exposures in four orientations and of the right coronary artery with two exposures in two orientations, as is routinely performed in our cardiology department. With one tube, the left coronary artery was imaged in the following orientations: posteroanterior, $30^{\circ}$ right anterior oblique with $20^{\circ}$ caudal angulation, and $15^{\circ}$ right anterior oblique with $30^{\circ}$ cranial angulation. With the other tube, the left coronary artery was imaged in the following orientations: $90^{\circ}$ left anterior oblique, $40^{\circ}$ left anterior oblique with $20^{\circ}$ cranial angulation, and $50^{\circ}$ left anterior oblique with $60^{\circ}$ caudal angulation. Angiography of the right coronary artery was performed in the following orientations: $30^{\circ}$ right anterior oblique and $15^{\circ}$ left anterior oblique with $15^{\circ}$ cranial angulation with one tube, and $60^{\circ}$ left anterior oblique and $90^{\circ}$ left anterior oblique with the other tube.
Additional views could be performed at the operator's discretion if necessary. In cases where a left ventricular angiogram was performed this part of radiation exposure was excluded. An experienced observer blinded to the results from CTCA evaluated the angiograms. Coronary arteries were included in the analysis if the luminal diameter was at least $1.5 \mathrm{~mm}$, excluding those vessels distal to complete occlusions. Each vessel was visually scored as being normal or significantly stenosed (defined as a diameter reduction of $\geqslant 50 \%$ ) reflecting daily clinical routine in our catheterisation laboratory.

\section{Effective radiation dose estimation $(E)$}

Values for $E$ were estimated for CTCA as a product of the doselength product $(\mathrm{DLP}) \times$ a conversion coefficient for the chest $(\mathrm{k}=0.017 \mathrm{mSv} / \mathrm{mGy} \times \mathrm{cm})$ as adapted by the European Commission in the European guidelines on quality criteria for computed tomography. ${ }^{18}$ Similarly, $E$ was estimated as a product of the dose-area product (DAP) of the diagnostic coronary scenes $\times$ a conversion factor for chest $(k=0.22 \mathrm{mSv} /$ $\mathrm{mGy} \times \mathrm{cm}^{2}$ ) for invasive CA based on the National Radiological Protection Board tables. ${ }^{19}$

\section{Statistical analysis}

Quantitative variables were expressed as mean (SD) and categorical variables or percentages. Man-Whitney U tests were performed for comparison of effective radiation dose between
Figure 3 Multiple calcified an uncalcified coronary lesions of an left descending artery are visualised on an angiographic view by CT coronary angiography $(A)$ and on an invasive coronary angiography image with matching angulations (B). The arrowhead indicates the most severe lesion in $(A)$ and $(B)$ as well as on the threedimensional volume rendered image (C). The mean effective radiation dose was $2.1 \mathrm{mSv}$ for CT and $7.3 \mathrm{mSv}$ for invasive coronary angiography.
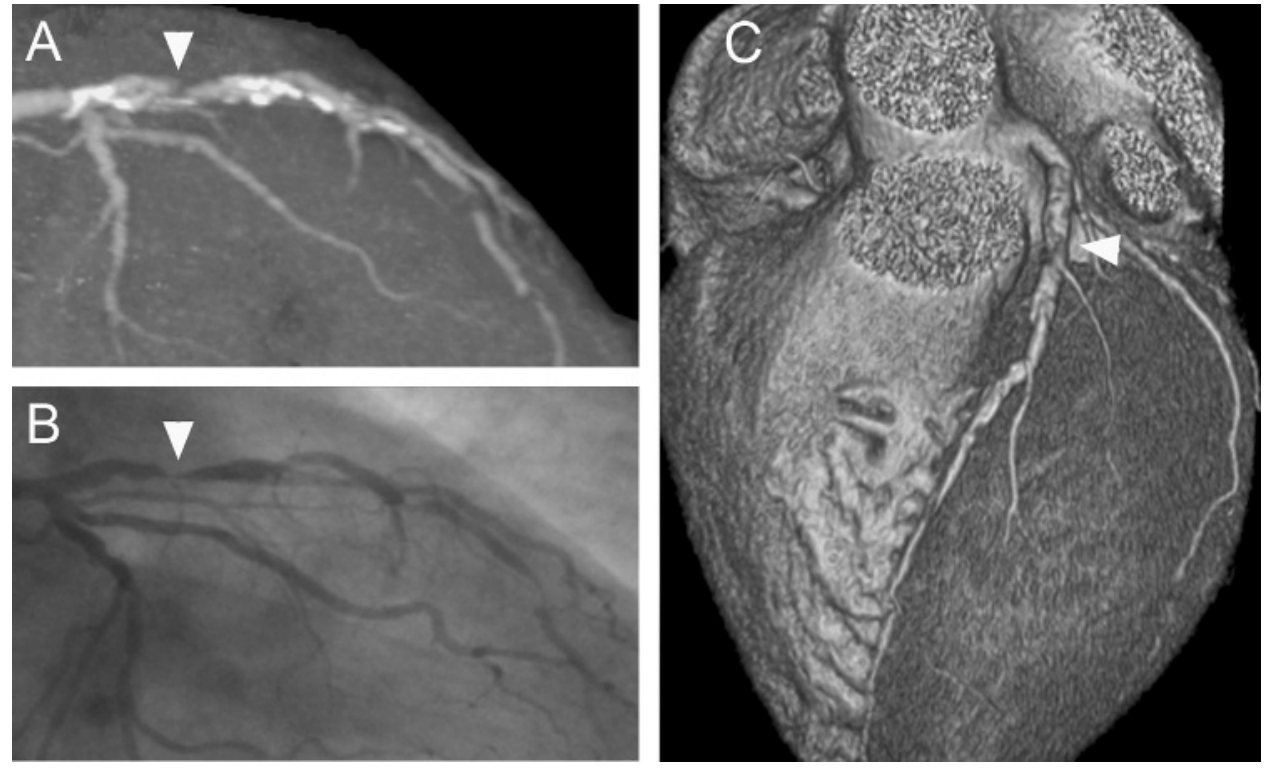
Figure 4 Left descending artery without coronary lesions is visualised on an angiographic view by CT coronary angiography $(A)$, on an invasive coronary angiography image with matching angulations $(B)$ and on the threedimensional volume rendered image (C). The mean effective radiation dose was $1.1 \mathrm{mSv}$ for CT and $5.9 \mathrm{mSv}$ for invasive coronary angiography.
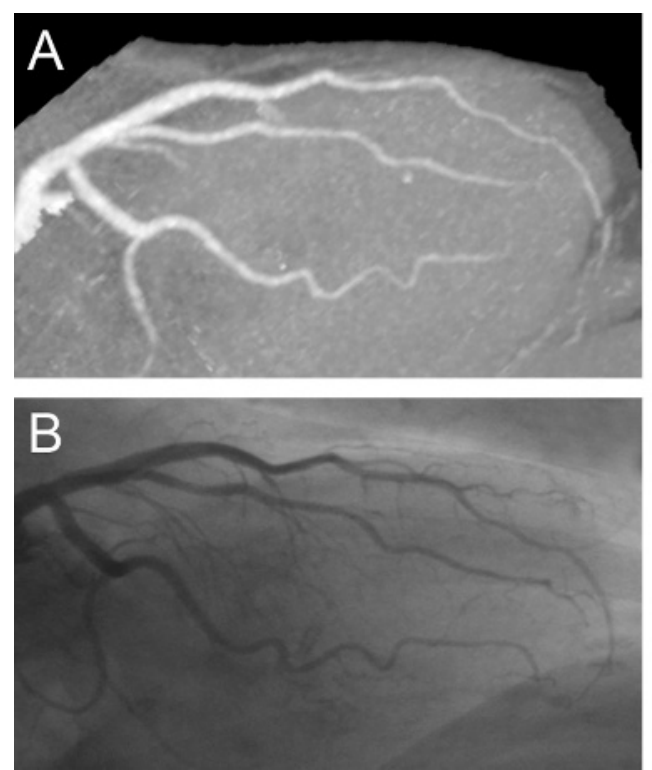

CTCA and invasive CA in all patients and different BMI subgroups by using SPSS software. A p value of $<0.0083$ was considered as significant using the Bonferroni-adjustment for multiple testing. Sensitivity, specificity, positive predictive value (PPV) and negative predictive value (NPV) were calculated from $\chi^{2}$ tests of contingency. The $95 \%$ confidence intervals (CI) and the positive and negative likelihood ratios (LR) were calculated.

\section{RESULTS}

\section{Effective radiation doses $(E)$}

For CTCA the mean DLP was 122.4 (42.1) $\mathrm{mGy} \mathrm{cm}$, resulting in a mean $E$ of $2.1(0.7) \mathrm{mSv}$. The variations of $E$ between all patients (DLP: $58.5-193.2 \mathrm{mGy} \mathrm{cm}$, E: $1.0-3.3 \mathrm{mSV}$ ) and within different BMI subgroups were small (fig 1 ).

For diagnostic invasive CA the DAP was 38.7 (4.4) $\mathrm{mGy} \mathrm{cm}^{2}$, resulting in a mean $E$ of 8.5 (4.4) $\mathrm{mSv}$. The doses varied widely between all patients (DAP: 6.4-93.0 mGy cm²; E: 1.4-20.5 mSv) and within different BMI subgroups (fig 1). There was no significant difference in $E$ of invasive CA between patients with stenoses (mean 9.4 (4.4) $\mathrm{mSv}$; range 1.4-20.5 mSv) and those with no CAD (mean 7.5 (4.3) $\mathrm{mSv}$, range 2.0-14.7 mSv).

Mean $E$ was significantly higher in invasive CA versus CTCA in all analysed patients $(p<0.001)$ (fig 2$)$. The mean DLP, DAP and $E$ for all patients and different BMI subgroups as well as their differences are given in table 1 .

\section{Image quality with CTCA}

Of 672 theoretically possible segments in 42 patients with 16 coronary segments, 64 were missing because of anatomical variants, 33 had a diameter of less than $1.5 \mathrm{~mm}$ at their origin (by both methods) and eight segments have been occluded. Thus, 64 segments were missing for reasons not associated with the methodology as these segments simply did not exist. The remaining 33 segments were categorised as lower than $1.5 \mathrm{~mm}$ and thus not evaluable by the gold standard invasive CA. Thus, the missing data do not seem to introduce a selection bias.

Image quality of $551 / 567$ segments (97.18\%) was diagnostic. Thus, 16 segments $(2.82 \%)$ were non-diagnostic and were considered as positive.

Diagnostic accuracy and impact of CTCA on pretest probability In $23(54.8 \%)$ patients and $52(31.0 \%)$ vessels, 80 coronary artery stenoses were recognised with invasive CA. Nineteen patients $(42.9 \%)$ had no CAD by invasive CA. Forty $(95.2 \%)$ patients have been correctly classified as having CAD (23/23) (example: fig 3) or no CAD (17/19) (example: fig 4).

On a per-vessel analysis, there was $94.2 \%$ (49/52; CI $0.8 \%$ to $1.0 \%)$ sensitivity, $94.8 \%(110 / 116 ; 95 \%$ CI $0.9 \%$ to $1.0 \%)$ specificity, $89.0 \%(49 / 55 ; 95 \%$ CI $0.8 \%$ to $1.0 \%)$ positive predictive value (PPV) and $97.4 \%$ (110/113; CI $0.9 \%$ to $1.0 \%)$ negative predictive value (NPV), yielding an accuracy of $94.6 \%$ (CI $09 \%$ to $1.0 \%$ ) (table 2 ).

Table 2 Diagnostic accuracy and predictive value for CTCA

\begin{tabular}{|c|c|c|c|c|c|c|c|c|c|c|c|c|c|}
\hline & $\begin{array}{l}\text { Pretest } \\
\text { probability }\end{array}$ & No & TP & FP & TN & FN & Sens & Spec & PPV & NPV & Accuracy & $+\mathrm{LR}$ & $-\mathbf{L R}$ \\
\hline Per patient & & 42 & 23 & 2 & 17 & 0 & 100 & 90 & 92 & 100 & 95 & 9.50 & 0 \\
\hline Low & 16 & 7 & 3 & 1 & 3 & 0 & 100 & 75 & 75 & 100 & 86 & 4.00 & 0 \\
\hline High & 87 & 13 & 7 & 1 & 5 & 0 & 100 & 83 & 100 & 88 & 92 & 6.00 & 0 \\
\hline Per vessel & - & 168 & 49 & 6 & 110 & 3 & 94 & 95 & 89 & 95 & 95 & 18.22 & 0.06 \\
\hline Low & - & 28 & 7 & 3 & 17 & 1 & 88 & 85 & 70 & 94 & 86 & 5.83 & 0.15 \\
\hline
\end{tabular}

FN, false negative; FP, false positive; LR, likelihood ratio; NA, not available; NPV, negative predictive value; PPV, positive predictive value; Sens, sensitivity; Spec, specificity; TN, true negative; TP, true positive. 
Figure 5 Impact of CT coronary angiography (CTCA) on pretest probabilities of significant coronary artery disease (CAD). 'Calculated using the Duke clinical score. ${ }^{2}$ Calculated using Bayesian statistics (post-test odds $=$ pretest odds $\times$ likelihood ratio).
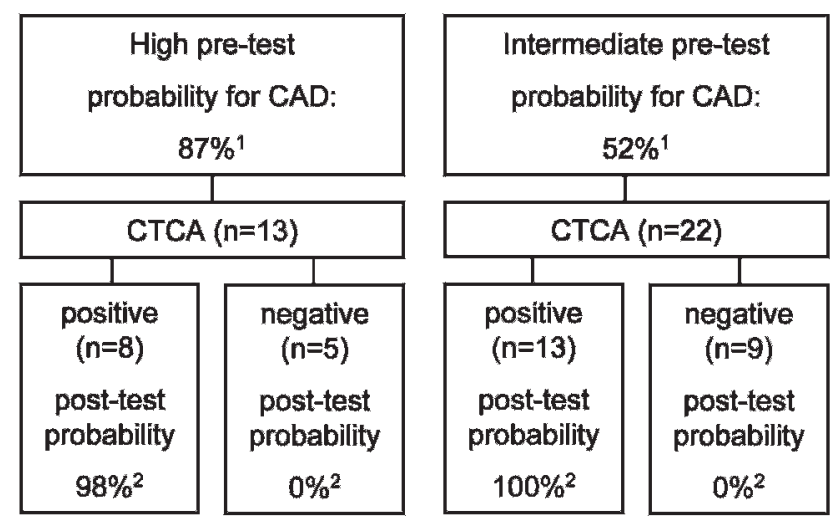

On a per-patient analysis, there was $100 \%$ (23/23; CI $0.9 \%$ to $-1.0 \%$ ) sensitivity, $89.5 \%$ (23/25; CI $0.7 \%$ to $1.0 \%)$ specificity, $92.0 \%(18 / 20,95 \%$ CI $0.7 \%$ to $1.0 \%)$ PPV and $100 \%(17 / 17$; CI $0.8 \%$ to $1.0 \%$ ) NPV, yielding an accuracy of $95.2 \%$ (CI $0.8 \%$ to $1.0 \%$ ) (table 2). The estimated pretest and post-test probabilities for significant CAD after CTCA scans are given in figure 5 .

\section{DISCUSSION}

While the gold standard for the detection of coronary lesions remains invasive CA, its associated costs, ${ }^{20}$ morbidity and mortality have driven the search for non-invasive alternatives like CTCA.

However, the radiation burden to patients has remained a concern and has led to hesitation to use non-invasive cardiac imaging with CT, although the introduction of tube modulation and optimised scanning parameters has allowed us to decrease $E$ of CTCA from over $20 \mathrm{mSv}$ to below $10 \mathrm{mSv}$ and may explain the large variability between different centres. ${ }^{8}$ New scanner technologies such as dual-source, ${ }^{21}$ as well as latest scanner generations with $256^{22}$ and more slices ${ }^{23}$ may allow the reduction of motions artefacts; the issue of radiation burden is not solved by these technical advances alone. In fact, in a preliminary study using 256 -slice radiation administered to the patient has been reported to be as high as $33 \mathrm{mSv}{ }^{22}$

As the strength of CTCA lies in its ability to exclude CAD (that is, its high NPV) the general perception is to use this technique mainly in low-to-intermediate probability populations. ${ }^{1}$ The latter however will not only have a low prevalence of CAD but inherently also bear a low risk of cardiovascular events, which makes it unlikely that any diagnostic procedure or therapeutic intervention would further improve the outcome. This may explain why it seems to be a particular challenge for CTCA to keep a positive balance of harms and benefits. It is in this context that the recently introduced scanning protocol using prospective ECG-triggering-where scanning is limited to a narrow predefined end-diastolic phase resulting in a massive reduction in radiation exposure ${ }^{11}{ }^{12}$ - was well received, as documented by a growing body of literature. ${ }^{13}{ }^{24-32}$ The present paper is the first to report on the head-to-head comparison of $E$ from invasive CA versus CTCA with prospective ECG-triggering in the same patients. Our results not only confirm the feasibility and reliability (accuracy versus invasive CA) of this low-dose CTCA protocol but also show that $E$ is significantly lower by about $75 \%$ than in CA. This holds true for each patient and is independent of the BMI and was not subject to a large inter-individual variability. By contrast, $E$ values from CA showed a large variability, in line with previous reports relating this variability to variances in procedure complexity, operator experiences or laboratory equipment. ${ }^{33}$ In fact, in patients with complex coronary anatomy such as diffuse wall alterations and multiple lesions with intermediate severity or those with bypass grafts $E$ from CA may increase considerably owing to the need for longer irradiation time, while the latter would not be affected using CTCA. Although in the present study we have excluded patients with known $\mathrm{CAD}$, thus eliminating patients with bypass grafts and reducing the probability of complex $\mathrm{CAD}$, we still found a significantly higher $E$ from CA with a substantial inter-individual variance as opposed to CTCA. This strengthens our data and indicates that the findings may remain valid even if extrapolated to various patient populations with different CAD probabilities.

As the same field of view was used in virtually all patients the $E$ from CTCA is mainly determined by the patient's physique because we used a BMI-adapted protocol for tube voltage and current. However, even after stratifying patients for BMI the variability of $E$ from CTCA was negligible while it remained high for CA.

Such a massive reduction in $E$ achieved by using prospective ECG-triggering of CTCA may have a profound impact on the perception of the optimal patient population benefiting most from non-invasive CTCA. It is an accepted rule that for reasons of radiation protection the total dose applied to a given population should be kept as low as reasonably achievable (ALARA). This dramatically shifts the benefit-to-harm balance supporting the hypothesis that low-dose CTCA may serve as a gatekeeper to CA in patient populations with CAD prevalence anywhere below 75\%. Outcome trials are needed to establish whether such a strategy may improve outcome. Theoretically, for each saved CA due to a normal CTCA (saved dose $6 \mathrm{mSv}$ ) we can afford three patients with CTCA plus CA (added dose of $2 \mathrm{mSv}$ compared to CA alone). The use of CTCA as gate keeper breaks even with regard to radiation exposure of the patient population with a prevalence of $75 \%$. The fact that the accuracy of CTCA does not reach $100 \%$ may negatively affect this balance. On the other hand preventing an unnecessary CA may confer more benefits than just reducing total radiation exposure as even elective purely diagnostic CA are associated with a low but not negligible morbidity and mortality. In addition, findings of coronary luminal narrowing often trigger coronary interventions that are associated with an even higher mortality despite no proved outcome benefits in chronic stable CAD patients. ${ }^{34}$ The values of $E$ clearly favour CTCA over CA, although the conversion factor for estimating $E$ from DLP in CA may be affected by a 10-20\% uncertainty. ${ }^{33}$ However, even with a conversion factor lower than the one used in our study the values remain significantly lower for CTCA. The advantage of 
prospective ECG-triggering as a new CTCA protocol to reduce $E$ lies in its universal applicability. In fact, this is not limited to the 64-slice CT scanners but can be implemented into the latest (dual-source) and future scanner generations such as those with 320 slices allowing full coverage of the heart in one rotation. The following technical limitations of CTCA have to be considered. Image quality is affected by coronary calcification, motion artefacts and body mass index. ${ }^{35}$ Furthermore, sinus rhythm and heart rate control are mandatory for prospective ECG-triggering, ${ }^{36}$ explaining the frequent $\beta$-blocker administration in this study. In addition, as the acquisition is limited to one phase the use of prospective ECG-triggering does not allow functional assessment of the left ventricle. Of note a substantial fraction of consenting patients had to be excluded for medical reasons. These contraindications, however, such as renal dysfunction or iodine allergy apply equally to spiral scanning and, therefore, do not represent specific limitations of prospective ECG-triggered CTCA. Moreover, in contrast to some other centres we do not routinely add unenhanced CT for calcium scoring to our CTCA, because there is no evidence supporting the idea that the additional radiation dose from calcium score is justified by an improvement in the accuracy of CTCA. ${ }^{1}$ Thus, we did not perform a calcium score scan in this study which contributed to the low radiation dose of the CTCA.

Although the relatively small population in this study may limit the power of the accuracy analysis for CTCA vs invasive CA this was not the primary goal of the study, which aimed at comparing the effective radiation dose of both techniques.

\section{CONCLUSIONS}

Low-dose CTCA allows evaluation of CAD with high accuracy, but a significantly less effective radiation dose to patients compared to diagnostic invasive CA.

Funding: The study was supported by a grant from the Swiss National Science Foundation (SNSF-professorship grant No PPO0A-114706) and by the ZIHP (Zurich Center for Integrative Human Physiology, University of Zurich, Switzerland).

Competing interests: None.

Ethics approval: The study is approved by the local research ethics committees.

Provenance and peer review: Not commissioned; externally peer reviewed.

\section{REFERENCES}

1. Schroeder S, Achenbach S, Bengel F, et al. Cardiac computed tomography: indications, applications, limitations, and training requirements: report of a Writing Group deployed by the Working Group Nuclear Cardiology and Cardiac CT of the European Society of Cardiology and the European Council of Nuclear Cardiology. Eur Heart J 2008;29:531-56.

2. Einstein AJ, Henzlova MJ, Rajagopalan S. Estimating risk of cancer associated with radiation exposure from 64-slice computed tomography coronary angiography. JAMA 2007:298:317-23.

3. Committee to Assess Health Risks from Exposure to Low Levels of lonizing Radiation; Nuclear and Radiation Studies Board DoEaLS, National Research Council of the National Academies. Health risks from exposure to low levels of ionizing radiation: BEIR VII Phase 2. Washington, DC: The National Academies Press, 2006.

4. Budoff MJ, Achenbach S, Blumenthal RS, et al. Assessment of coronary artery disease by cardiac computed tomography: a scientific statement from the American Heart Association Committee on Cardiovascular Imaging and Intervention, Council on Cardiovascular Radiology and Intervention, and Committee on Cardiac Imaging, Council on Clinical Cardiology. Circulation 2006;114:1761-91.

5. Brenner DJ, Elliston CD. Estimated radiation risks potentially associated with fullbody CT screening. Radiology 2004;232:735-8.

6. Mollet NR, Cademartiri F, van Mieghem CA, et al. High-resolution spiral computed tomography coronary angiography in patients referred for diagnostic conventional coronary angiography. Circulation 2005;112:2318-23.

7. Leber AW, Knez A, von Ziegler F, et al. Quantification of obstructive and nonobstructive coronary lesions by 64-slice computed tomography: a comparative study with quantitative coronary angiography and intravascular ultrasound. J Am Coll Cardiol 2005;46:147-54.

8. Stolzmann P, Scheffel H, Schertler T, et al. Radiation dose estimates in dual-source computed tomography coronary angiography. Eur Radiol 2008;18:592-9.
9. Hsieh J, Londt J, Vass M, et al. Step-and-shoot data acquisition and reconstruction for cardiac x-ray computed tomography. Med Phys 2006;33:4236-48.

10. Earls JP, Berman EL, Urban BA, et al. Prospectively gated transverse coronary CT angiography versus retrospectively gated helical technique: improved image quality and reduced radiation dose. Radiology 2008;246:742-53.

11. Husmann L, Valenta I, Gaemperli 0, et al. Feasibility of low-dose coronary CT angiography: first experience with prospective ECG-gating. Eur Heart J 2008;29:191-7.

12. Herzog BA, Husmann L, Burkhard N, et al. Accuracy of low-dose computed tomography coronary angiography using prospective electrocardiogram-triggering: first clinical experience. Eur Heart J 2008;29:3037-42.

13. Maruyama T, Takada M, Hasuike T, et al. Radiation dose reduction and coronary assessability of prospective electrocardiogram-gated computed tomography coronary angiography comparison with retrospective electrocardiogram-gated helical scan. J Am Coll Cardiol 2008;52:1450-5.

14. United Nations. Sources and effects of ionizing radiation: United Nations Scientific Committee on the Effects of Atomic Radiation UNSCEAR 2000 Report to the General Assembly, with Scientific Annexes. New York: UN, 2000.

15. Gibbons RJ, Balady GJ, Bricker JT, et al. ACC/AHA 2002 guideline update for exercise testing: summary article: a report of the American College of Cardiology/ American Heart Association Task Force on Practice Guidelines (Committee to Update the 1997 Exercise Testing Guidelines). Circulation 2002;106:1883-92.

16. Leschka S, Wildermuth S, Boehm T, et al. Noninvasive coronary angiography with 64-section CT: effect of average heart rate and heart rate variability on image quality. Radiology 2006;241:378-85.

17. Austen WG, Edwards JE, Frye RL, et al. A reporting system on patients evaluated for coronary artery disease. Report of the Ad Hoc Committee for Grading of Coronary Artery Disease, Council on Cardiovascular Surgery, American Heart Association. Circulation 1975;51:5-40.

18. Commission E. European guidelines on quality criteria for computed tomography, EUR 16262EN. Luxembourg: Office for Official Publications of the European Communities, 2000. Available at:http://www.drs.dk/guidelines/ct/quality/htmlindex. htm. (Accessed 21 November 2008.)

19. Lobotessi H, Karoussou A, Neofotistou V, et al. Effective dose to a patient undergoing coronary angiography. Radiat Prot Dosimetry 2001;94:173-6.

20. Scanlon PJ, Faxon DP, Audet AM, et al. ACC/AHA guidelines for coronary angiography. A report of the American College of Cardiology/American Heart Association Task Force on practice guidelines (Committee on Coronary Angiography). Developed in collaboration with the Society for Cardiac Angiography and Interventions. J Am Coll Cardiol 1999;33:1756-824.

21. Leschka S, Alkadhi H, Plass A, et al. Accuracy of MSCT coronary angiography with 64-slice technology: first experience. Eur Heart J 2005;26:1482-7.

22. Motoyama S, Anno H, Sarai M, et al. Noninvasive coronary angiography with a prototype 256-row area detector computed tomography system: comparison with conventional invasive coronary angiography. J Am Coll Cardiol 2008;51:773-5.

23. Rybicki FJ, Otero HJ, Steigner ML, et al. Initial evaluation of coronary images from 320-detector row computed tomography. Int J Cardiovasc Imaging 2008;24:535-46.

24. Henneman MM, Schuijf JD, Pundziute G, et al. Noninvasive evaluation with multislice computed tomography in suspected acute coronary syndrome: plaque morphology on multislice computed tomography versus coronary calcium score. J Am Coll Cardiol 2008;52:216-22

25. Kaufmann PA. Low-dose computed tomography coronary angiography with prospective triggering a promise for the future. J Am Coll Cardiol 2008:52:1456-7.

26. Le Polain de Waroux JB, Pouleur AC, Goffinet C, et al. Combined coronary and late-enhanced multidetector-computed tomography for delineation of the etiology of left ventricular dysfunction: comparison with coronary angiography and contrastenhanced cardiac magnetic resonance imaging. Eur Heart J 2008;29:2544-51.

27. Lindsay AC, Choudhury RP. Form to function: current and future roles for atherosclerosis imaging in drug development. Nat Rev Drug Discov 2008;7:517-29.

28. Pundziute G, Schuijf JD, Jukema JW, et al. Evaluation of plaque characteristics in acute coronary syndromes: non-invasive assessment with multi-slice computed tomography and invasive evaluation with intravascular ultrasound radiofrequency data analysis. Eur Heart J 2008;29:2373-81.

29. Rixe J, Rolf A, Conradi G, et al. Image quality on dual-source computed-tomographic coronary angiography. Eur Radiol 2008;18:1857-62.

30. Rybicki FJ, Otero HJ, Steigner ML, et al. Initial evaluation of coronary images from 320-detector row computed tomography. Int J Cardiovasc Imaging 2008;24:535-46.

31. Scheffel H, Alkadhi H, Leschka S, et al. Low-dose CT coronary angiography in the step-and-shoot mode: diagnostic performance. Heart 2008;94:1132-7.

32. Schmermund A, Nowak B, Voigtlander T. Non-invasive CT coronary angiography: can high diagnostic image quality be achieved with less radiation exposure? Eur Heart J 2008;24:2955-6.

33. Einstein AJ, Moser KW, Thompson RC, et al. Radiation dose to patients from cardiac diagnostic imaging. Circulation 2007;116:1290-305.

34. Boden WE, O'Rourke RA, Teo KK, et al. Optimal medical therapy with or without PCI for stable coronary disease. N Engl J Med 2007;356:1503-16

35. Tatsugami F, Husmann L, Herzog BA, et al. Evaluation of a body mass index-adapted protocol for low-dose 64-MDCT coronary angiography with prospective ECG triggering. AJR Am J Roentgenol 2009;192:635-8.

36. Herzog BA, Husmann L, Burkhard N, et al. Low-dose CT coronary angiography using prospective ECG-triggering: impact of mean heart rate and heart rate variability on image quality. Acad Radiol 2009;16:15-21. 